\title{
Active dynamics of filaments in motility assays
}

\author{
J. Kierfeld ${ }^{1, a}$, K. Frentzel ${ }^{1}$, P. Kraikivski ${ }^{2}$, and R. Lipowsky ${ }^{1}$ \\ 1 Max-Planck-Institute of Colloids and Interfaces, Science Park Golm, 14424 Potsdam, Germany \\ 2 University of Connecticut Health Center, 263 Farmington Avenue, Farmington, Connecticut 06030, \\ USA
}

\begin{abstract}
We study the active dynamics of single and interacting cytoskeletal filaments in motility assays, in which immobilized motor proteins bind the filaments to a surface and actively pull them along this surface. We present a model which couples the overdamped dynamics of filaments, the active dynamics of motor heads, and the elasticity of motor stalks and which can be used for Langevin dynamics simulations. Single filaments perform a persistent random walk, which we characterize by several simulation results. For interacting filaments with a repulsive interaction of filaments, the motor-driven dynamics of filaments leads to a non-equilibrium phase transition which generalizes the isotropic-nematic phase transition of the corresponding equilibrium system, the hard-rod fluid. Langevin dynamics simulations and analytical theory show that the motor activity enhances the tendency for nematic ordering.
\end{abstract}

\section{Introduction}

The cytoskeleton of eucaryotic cells is made up by filaments, which are semiflexible polymers [1]. The cytoskeleton of a living cell plays an important role for cell division, cell motility, and force generation $[2,3]$. These processes require the cytoskeleton to be a dynamic structure, which can constantly re-organize itself. For this reason, the dynamics of cytoskeletal filaments involves several active processes, which are driven by the coupling to the hydrolysis of nucleotide triphosphates (NTPs) such as ATP or GTP. Two important NTP-driven processes are the polymerization dynamics, where NTP-hydrolysis allows for treadmilling and force generation by polymerizing filaments, and the actuation of filaments by motor proteins, which generate small forces in the piconewton range. Whereas the conventional dynamics of polymers is governed by thermal fluctuations [4], such motor forces give rise to an active filament dynamics, which is driven by a constant supply of mechanical energy through ATP-hydrolysis in motor proteins.

In order to understand the underlying principles of motor-driven filament dynamics it is necessary to study simple model systems in vitro. One such model system, which has been intensively studied, are bulk solutions of microtubules and two-headed kinesin motor proteins [5-7], in which the formation of patterns such as asters and vortices is observed. Theoretical studies of such patterns [8-12] have used a coarse-grained continuum description with kinetic equations for filament density and orientation fields and a motor-density field. In these approaches it is inherently difficult to relate the macroscopic transport coefficients to the experimentally accessible microscopic parameters of the system. Based on experimental work on actin solutions containing myosin motor mini-filaments [13] it has also been suggested that the effect of motor activity can be described by an increased effective temperature in the non-equilibrium system [14].

In this article, the dynamics and pattern formation by filaments in motility assays is investigated. In a motility assay the tails of motor proteins are adsorbed and anchored to a

\footnotetext{
a e-mail: Jan.Kierfeld@mpikg.mpg.de
} 

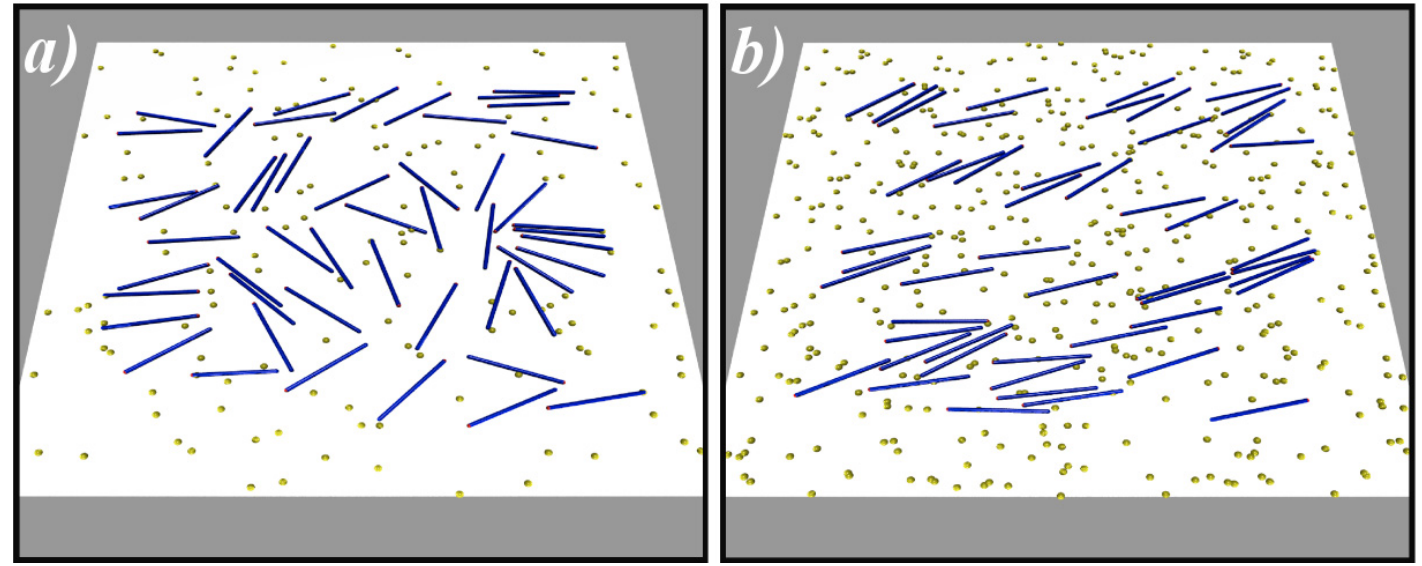

Fig. 1. Snapshots of rodlike filaments with hard-core repulsion on a motor coated substrate with randomly distributed motors and periodic boundary conditions. The filament concentration is $\rho=2 / L^{2}$, i.e., below the critical concentration of the equilibrium isotropic-nematic transition. For detachment forces $F_{d}=F_{s t}$, we find (a) an isotropic phase at low motor surface density $\sigma \ell_{m} L=0.03$ and (b) active nematic ordering at high motor surface density $\sigma \ell_{m} L=0.09$.

two-dimensional surface. The filaments are pulled over this surface if the molecular motors are active. Because of their reduced dimensionality and the quenched motor concentration field, motility assays represent a slightly simpler model system in comparison to systems consisting of solutions of filaments and two-headed motor proteins. Motility or gliding assays are by now a standard tool to characterize motor proteins by analyzing the transport velocities of single filaments gliding over the substrate $[15,16]$. We will first discuss the dynamics of single filaments. One important quantity characterizing the motion of a single filament is the mean velocity of filaments. Single filaments perform a persistent random walk [17-19], which we characterize using our simulation model. Then we focus on the cooperative behavior of many filaments, which have a mutual repulsive interaction [20,21]. Repulsive interactions originate from the steric interaction of filaments, which gives rise to an additional bending energy cost associated with each crossing of two filaments. This crossing energy cost can become large if the filaments are effectively confined to two dimensions by decreasing the height in the direction perpendicular to the surface below two filament diameters. In this case, a hard-core repulsion between filaments is effective. The equilibrium system corresponding to the confined motility assay with hard-core repulsion in the absence of motors is the two-dimensional hard-rod fluid, which exhibits an isotropic-nematic ordering transition above a critical density of filaments [22,23]. We show both numerically using a microscopic simulation model and by analytical arguments that the nematic ordering is enhanced by the presence of motor activity due to the combined effect of repulsive filament interactions and active forces exerted by the motors; see Fig. 1. Using the results for the persistent motion of single filaments we extend the dynamic mean-field theory for nematic ordering [24] to active systems. From the theoretical treatment, we derive the concept of an increased effective length, which successfully explains our simulation data for filaments with a hard-core repulsion characterized by an infinite filament crossing energy and allows to obtain the phase behavior in terms of the experimentally accessible microscopic model parameters. Our results show that the concept of an effective temperature is rather subtle and does not apply in the limit of large crossing energy barriers where the system becomes effectively athermal, i.e., is governed by steric interactions only.

\section{Model}

We first introduce our microscopic model for filament motion in motility assays. The model contains filaments, motor heads, and polymeric motor tails as separate degrees of freedom, see 


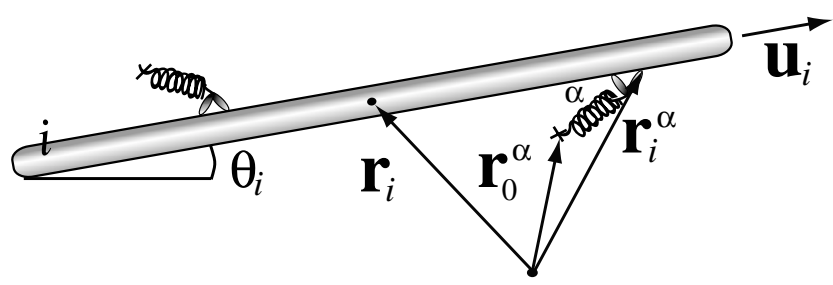

Fig. 2. Schematic top view of a filament $i$ in the motility assay with two motors attached. $\mathbf{r}_{i}$ is the filament's center of mass, $\theta_{i}$ and $\mathbf{u}_{i}$ its orientational angle and unit vector, respectively. The attached motor $\alpha$ is anchored at $\mathbf{r}_{0}^{\alpha}$, and its head is positioned at $\mathbf{r}_{i}^{\alpha}$. Motor stalks or tails connecting the anchor points and the motor heads are modeled as polymeric springs.

Fig. 2. One end of the motor tail is anchored to the substrate, and the motor head on the other end can bind to a filament. We assume that the tail flexibility allows for binding in the correct orientation with respect to the filament polarity. Once bound, the motor head moves along the filament thereby stretching the polymeric motor tail, which gives rise to a loading force acting both on the motor head and the attached filament. This force feeds back onto the motion of the bound motor head, which moves with a load-dependent motor velocity $[25,26]$. Filaments follow an overdamped dynamics with external forces arising from the stretched motor tails and from the hard-core repulsion between filaments.

To proceed, let us consider $N$ rigid filaments of length $L$ (with index $i=1, \ldots, N$ ) on a planar two-dimensional surface. The configuration of filament $i$ can then be specified by the two-dimensional vector $\mathbf{r}_{i}$ for its center of mass and by the angle $\theta_{i}$ or the unit vector $\mathbf{u}_{i}=$ $\left(\cos \theta_{i}, \sin \theta_{i}\right)$ for its orientation; see Fig. 2. The filament is subject to forces $\mathbf{F}_{i}^{\alpha}$ from $N_{i}$ attached motors (with index $\alpha=1, \ldots, N_{i}$ ) with motor heads positioned at $\mathbf{r}_{i}^{\alpha}$. Each such force arises from the polymeric tail of motor $\alpha$, which is stretched by the directed motion of the motor head on the filament, as described below. The motor tail is anchored at $\mathbf{r}_{0}^{\alpha}$ and the head position is $\mathbf{r}_{i}^{\alpha}$. We model the polymeric tail of the motor as a freely jointed chain such that $\mathbf{F}_{i}^{\alpha}$ is obtained by inverting the force-extension relation of a freely jointed chain. ${ }^{1}$ In addition to motor forces, the filaments are subject to interaction forces $\mathbf{F}_{i j}$ arising from the purely repulsive interactions between filaments $i$ and $j[28]$.

Under the influence of the motor forces $\mathbf{F}_{i}^{\alpha}$ and the interaction forces $\mathbf{F}_{i j}$ each filament $i$ performs an overdamped translational motion, which is described by the stochastic Langevintype equation of motion [20]

$$
\boldsymbol{\Gamma} \cdot \partial_{t} \mathbf{r}_{i}=\sum_{\alpha=1}^{N_{i}} \mathbf{F}_{i}^{\alpha}+\sum_{j=1}^{N} \mathbf{F}_{i j}+\boldsymbol{\zeta}_{i}
$$

where

$$
\boldsymbol{\Gamma} \equiv \Gamma_{\|} \mathbf{u}_{i} \otimes \mathbf{u}_{i}+\Gamma_{\perp}\left(\mathbf{I}-\mathbf{u}_{i} \otimes \mathbf{u}_{i}\right),
$$

is the matrix of translational friction coefficients, which contains friction coefficients $\Gamma_{\|}$and $\Gamma_{\perp}$ for motion parallel and perpendicular to the filament orientation $\mathbf{u}_{i}$, respectively, and $\boldsymbol{\zeta}_{i}(t)$ are the Gaussian distributed thermal random forces [4]. In eq. (2), I is the unit matrix and $\otimes$ the dyadic vector product. The thermal noise $\boldsymbol{\zeta}_{i}(t)$ has correlations

$$
\left\langle\boldsymbol{\zeta}_{i}(t) \otimes \boldsymbol{\zeta}_{j}\left(t^{\prime}\right)\right\rangle=2 T \boldsymbol{\Gamma} \delta_{i j} \delta\left(t-t^{\prime}\right) .
$$

We measure the temperature in energy units, i.e., $k_{B} \equiv 1$.

In addition to filament translation, motor and interaction forces give also rise to the torques $M_{i}^{\alpha} \equiv\left|\left(\mathbf{r}_{i}^{\alpha}-\mathbf{r}_{i}\right) \times \mathbf{F}_{i}^{\alpha}\right|$ and $M_{i j}$, respectively [28]. These torques lead to an overdamped rotational dynamics, which is described by

$$
\Gamma_{\theta} \partial_{t} \theta_{i}=\sum_{\alpha=1}^{N_{i}} M_{i}^{\alpha}+\sum_{j=1}^{N} M_{i j}+\zeta_{\theta, i}
$$

${ }^{1}$ If the motor tail is anchored at $\mathbf{r}_{0}^{\alpha}$, the force $-\mathbf{F}_{i}^{\alpha}$ is pointing in the direction $\Delta \mathbf{r}^{\alpha} \equiv \mathbf{r}_{i}^{\alpha}-\mathbf{r}_{0}^{\alpha}$ and $\left|\Delta \mathbf{r}^{\alpha}\right| / L_{m}=f_{\mathrm{FJC}}\left(\left|\mathbf{F}_{i}^{\alpha}\right| b_{m} / T\right)$, where $L_{m}$ is the contour and $b_{m}$ the monomer length of the polymeric motor tail, $k_{B} \equiv 1$, and $f_{\mathrm{FJC}}(x) \equiv 1 / \tanh x-1 / x$, cf. Ref. [27]. 
where $\Gamma_{\theta}$ is the rotational friction coefficient and $\zeta_{\theta, i}(t)$ is a Gaussian distributed thermal random torque. The thermal torque $\zeta_{\theta, i}(t)$ has correlations

$$
\left\langle\zeta_{\theta, i}(t) \zeta_{\theta, j}\left(t^{\prime}\right)\right\rangle=2 T \Gamma_{\theta} \delta_{i j} \delta\left(t-t^{\prime}\right) .
$$

Note that all friction coefficients $\Gamma_{\|}, \Gamma_{\perp}$ and $\Gamma_{\theta}$ are identical to those of the passive filament dynamics, see Ref. [4].

The dynamics of motor heads is described by a deterministic equation of motion

$$
\partial_{t} x_{i}^{\alpha}=v\left(\mathbf{F}_{i}^{\alpha}\right)
$$

where $\left|x_{i}^{\alpha}\right| \leq L / 2$ defines the position of the motor $\alpha$ along the $\operatorname{rod} i$, i.e., $\mathbf{r}_{i}^{\alpha}=\mathbf{r}_{i}+x_{i}^{\alpha} \mathbf{u}_{i}$, and the filament polarity is such that the motor head moves in the direction $\mathbf{u}_{i}$. The motor velocity $v$ is a function of the loading force $\mathbf{F}_{i}^{\alpha}$ which builds up due to stretching of the motor tail. We use a force-velocity relation with a maximum value $v_{\max }$ for forces $\mathbf{F}_{i}^{\alpha} \cdot \mathbf{u}_{i} \geq 0$ pulling the motor forward, a linear decrease for forces $\mathbf{F}_{i}^{\alpha} \cdot \mathbf{u}_{i}<0$ pulling the motor backwards, and $v=0$ for $\mathbf{F}_{i}^{\alpha} \cdot \mathbf{u}_{i}<-F_{s t}$, where $F_{s t}$ is the stall force $[25,26]$.

We assume that the motor binds to the filament when the distance between the anchored end of the motor tail at $\mathbf{r}_{0}^{\alpha}$ and (the middle line of) the filament is smaller than a capture radius $\ell_{m}$. Apart from the stall force $F_{s t}$ the motor is also characterized by its detachment force $F_{d}$, above which the unbinding rate of the motor head becomes large. For simplicity we assume in our model that the motor head detaches whenever the force $F_{i}^{\alpha}$ exceeds a threshold value $F_{d}$. We consider the case of processive motors with a high duty ratio close to unity, i.e., motors detach from a filament only if they reach the filament end or if the force $F_{i}^{\alpha}$ becomes larger than the detachment force $F_{d}$.

\section{Simulation}

Using the above model we performed simulations of gliding assays for a uniform random distribution of motors with a surface density $\sigma$ and periodic boundary conditions. At each time step $\Delta t$, we update the motor head positions $x_{i}^{\alpha}$ and filament positions and orientations using the discretized versions of the equations of motions described above. Each data point in the phase diagram in Fig. 5 and Fig. 6 below corresponds to simulation runs over $10^{6}$ time steps. In the simulations we take time steps $\Delta t=10^{-3} \mathrm{~s}$ such that $10^{6}$ time steps correspond to $10^{3} \mathrm{~s}$.

The parameter values that we choose for the simulations are comparable with experimental data on assays for conventional kinesin. The simulation results presented in Figs. 1 and 5 have been obtained for quadratic assays of area $25 \mu \mathrm{m}^{2}$ with filaments of length $L=1 \mu \mathrm{m}$ and diameter $D=L / 40$ at room temperature $T \simeq 4 \times 10^{-3} \mathrm{pN} \mu \mathrm{m}$. The friction coefficients are taken to be $\Gamma_{\perp}=2 \Gamma_{\|}=4 \pi \eta L / \ln (L / D)$ and $\Gamma_{\theta}=\Gamma_{\|} L^{2} / 6$, where $\eta$ is the viscosity of the surrounding liquid [4]. We use a value $\eta=0.5 \mathrm{pN} \mathrm{s} / \mu \mathrm{m}^{2}$ much higher than the viscosity of water, $\eta_{\text {water }} \sim 10^{-3} \mathrm{pNs} / \mu \mathrm{m}^{2}$, which allows to take larger simulation time steps. The ratio $\Delta t / \eta$ is chosen sufficiently small that motor forces that build up during a single time step remain comparable to frictional forces, which makes the simulation numerically stable. We checked that our choice of viscosity does not affect results by performing selected simulation runs also at the viscosity of water. We use a maximum motor speed of $v_{\max }=1 \mu \mathrm{m} / \mathrm{s}$ and a stall force of $F_{s t}=5 \mathrm{pN}$. The capture radius for motor proteins is chosen as $\ell_{m}=22 \mathrm{~nm}$ for the simulations presented in section 4 and $\ell_{m}=10 \mathrm{~nm}$ for the simulations in section 5 . For the length of the fully stretched polymeric motor tail we take $L_{m}=50 \mathrm{~nm}$. For the simulations in sections 4 and 5 we use monomer lengths of $b_{m}=10 \mathrm{~nm}$ and $b_{m}=4 \mathrm{~nm}$, respectively, in the motor tail.

Our simulation model differs from previous microscopic simulation models $[17,19]$ in several respects. In Ref. [17] the polymeric motor stalks are not modeled explicitly. Therefore, filament motion perpendicular to the filaments' orientation and filament rotation are completely suppressed while more than one motor is attached. As we will discuss in the next section this suppresses a fluctuating component of the filament motion in the direction perpendicular to its orientation, which is caused by the stepping of attached motors if more than one motor is 
attached, by attachment and detachment of motors, and by thermal forces. Our model differs from the model used in Ref. [19] for the simulation of single filaments because we use a more detailed model of the elasticity of the polymeric motor stalks. Moreover, we include additional forces between the filaments.

\section{Dynamics of single filaments}

The dynamics of single filaments in the motility assay depends strongly on the number of motors which are attached to and actively driving the filament. This directly influences the mean velocity $v_{F}$ of the filament and the distance $\xi_{\text {rot }}$ it travels between successive rotations.

The number of motors $N_{i}$ which is attached to a filament $i$ is related to the distance between these bound motors. The mean distance between bound motors $\left\langle d_{m}\right\rangle$ depends on the length of the filament and the motor surface density. For long filaments or high motor density, we find $\left\langle d_{m}\right\rangle \approx 1 / 2 \sigma \ell_{m}$. In this limit $\left\langle d_{m}\right\rangle$ is determined by the condition that the probability to find a motor within the area $2\left\langle d_{m}\right\rangle \ell_{m}$ is equal to one because motors can attach to the filament from a distance $\leq \ell_{m}$. This argument is correct as long as the filament length $L$ is much larger than the mean motor separation $\left\langle d_{m}\right\rangle$, i.e., for $\sigma \ell_{m} L \gg 1$. In this regime many motors are attached to a single filament. For short filaments or low motor density $\sigma \ell_{m} L \ll 1$, on the other hand, the mean distance between bound motors cannot become smaller than the filament length, and we expect $\left\langle d_{m}\right\rangle \sim L$. Both results can be combined into the approximate formula

$$
\left\langle d_{m}\right\rangle \approx \frac{L}{1+2 \sigma \ell_{m} L} .
$$

We consider the limit of high motor surface density $\sigma \ell_{m} L \gg 1$, and let the filament $i$ advance by a distance $L$ of its own length. Then it looses all previously attached motors and acquires a completely new set of motors. Motors attach with a constant probability $1 /\left\langle d_{m}\right\rangle=1 / 2 \sigma \ell_{m}$ per advanced length $\Delta L$. Then the attachment of motors is governed by a Poisson process and the probability $P\left(N_{i}\right)$ to find $N_{i}$ motors on the filament after advancing the distance $L$ is given by the Poisson distribution

$$
P\left(N_{i}\right)=\frac{1}{N_{i} !}\left(\frac{L}{\left\langle d_{m}\right\rangle}\right)^{N_{i}} e^{-L /\left\langle d_{m}\right\rangle}
$$

and the mean number of attached filaments is

$$
\left\langle N_{i}\right\rangle \approx \frac{L}{\left\langle d_{m}\right\rangle} \approx 2 \sigma \ell_{m} L .
$$

In the limit of high motor surface density $\sigma \ell_{m} L \gg 1$ which we consider, we have $\left\langle N_{i}\right\rangle \gg 1$, i.e., many motors are attached to a filament $i$.

One important quantity, which can be directly measured in a motility assay is the mean filament velocity $v_{F}$ of a filament if it is attached to and driven by at least one motor. For a single filament $i$ the mean velocity is given by $v_{F}=\left|\left\langle\partial_{t} \mathbf{r}_{i}\right\rangle\right|$. In a steady state motion where the filament is pulled by one or several motors, the mean filament velocity can be obtained by simultaneously equating (i) the filament friction force with the total motor driving force parallel to the filament and (ii) the filament velocity with the motor velocity in the steady state. This estimate will involve the mean-field-like approximation that all motors $\alpha$ attached to a filament $i$ have the same average velocity $\left\langle\partial_{t} x_{i}^{\alpha}\right\rangle$ and that we can neglect correlations between different motors. The mean number of attached motors is $\left\langle N_{i}\right\rangle \approx L /\left\langle d_{m}\right\rangle$ according to (9). Each motor pulls with an average force $\left\langle F_{i, \|}^{\alpha}\right\rangle \equiv\left\langle\mathbf{F}_{i}^{\alpha} \cdot \mathbf{u}_{i}\right\rangle$ in the direction parallel to the filament. This gives a total motor driving force $\left\langle N_{i}\right\rangle\left\langle F_{i, \|}^{\alpha}\right\rangle$, where we assumed that fluctuations of both quantities are uncorrelated. Equating this with the filament friction force in the direction parallel to the filament, we find

$$
\Gamma_{\|} v_{F}=\left\langle N_{i}\right\rangle\left\langle F_{i, \|}^{\alpha}\right\rangle
$$


The mean velocity of a motor loaded by a mean force $\left\langle F_{i, \|}^{\alpha}\right\rangle$ in the direction parallel to the filament is

$$
\left\langle\partial_{t} x_{i}^{\alpha}\right\rangle=v_{\max }\left(1-\left\langle F_{i, \|}^{\alpha}\right\rangle / F_{s t}\right),
$$

where we assumed that the non-linear regime of the force-velocity relation is not relevant in the steady state. In the steady state the mean velocity of each attached motor has to be equal to the mean filament velocity,

$$
v_{F}=\left\langle\partial_{t} x_{i}^{\alpha}\right\rangle .
$$

Combining all three equations (10), (11), and (12) we find for the inverse average total motor force the relation

$$
\frac{1}{\left\langle N_{i}\right\rangle\left\langle F_{i, \|}^{\alpha}\right\rangle}=\frac{1}{\left\langle N_{i}\right\rangle F_{s t}}+\frac{1}{\Gamma_{\|} v_{\max }},
$$

i.e., the average total force is the harmonic mean of the total average motor force and the maximal friction force, which also shows that $0<\left\langle F_{i, \|}^{\alpha}\right\rangle\left\langle F_{s t}\right.$ and, indeed, the motors operate in the regime of a linear force-velocity relation. The corresponding velocity of the filament is given by

$$
\frac{1}{v_{F}}=\frac{\Gamma_{\|}}{\left\langle N_{i}\right\rangle F_{s t}}+\frac{1}{v_{\max }}
$$

We can distinguish between a regime of strong motor forces for $\left\langle N_{i}\right\rangle F_{s t} \gg \Gamma_{\|} v_{\max }$ and weak motor forces for $\left\langle N_{i}\right\rangle F_{s t} \ll \Gamma_{\|} v_{\max }$. Typical values for kinesin give $\Gamma_{\|} v_{\max } / F_{\text {st }} \sim$ $(L / \mu \mathrm{m}) / \ln (L / D)$ such that filaments much longer than $1 \mu \mathrm{m}$ experience strong motor forces if $\left\langle N_{i}\right\rangle>1$. This is the typical situation for motility assays of microtubules and kinesin [15]. In the limit of strong motor forces, the velocity of the driven filament is limited by the maximal motor velocity, $v_{F} \approx v_{\max }$. This leads to a filament velocity which is independent of the motor density as observed for assays of microtubules and kinesin in Ref. [15]. In the limit of weak motor forces, on the other hand, the viscous drag dominates and the filament velocity becomes small, $v_{F} \ll v_{\max }$.

The motion of a filament is characterized by stochastic switching between three modes of motion: rotational and translational diffusion if no motors are attached, directed translation in rotationally diffusing directions if one motor is attached, and directed translation if two or more motors are attached. The directed translation proceeds with the velocity $v_{F}$ determined by eq. (14). The relative frequency of these types of motion depends on the mean distance $\left\langle d_{m}\right\rangle$ between bound motors and, thus, on the surface motor concentration $\sigma$, see (7). The distance $\xi_{\text {rot }}$ traveled by a filament between successive rotations is given by the the first passage time from a state with two motors attached to a state with a single motor attached. The mean distance $\left\langle\xi_{\text {rot }}\right\rangle$ between rotations has been calculated in Ref. [17]. The resulting motion of a single filament alternates between straight motion over average distances $\left\langle\xi_{\text {rot }}\right\rangle$ and rotational diffusion about an angle $\langle|\Delta \theta|\rangle=3 / \sigma L^{2}$. This motion is a persistent random walk with a persistence length $[17]$

$$
\xi_{p}=\frac{\left\langle\xi_{\text {rot }}\right\rangle}{\langle|\Delta \theta|\rangle^{2}}=\frac{L+2\left\langle d_{m}\right\rangle}{L+3\left\langle d_{m}\right\rangle} \frac{L^{3} \sigma^{2}\left\langle d_{m}\right\rangle^{2}}{9}\left(e^{L /\left\langle d_{m}\right\rangle}-1-\frac{L}{\left\langle d_{m}\right\rangle}\right) .
$$

These theoretical predictions can be checked using our microscopic simulation model. As a first step we can visualize the motion of a single filament in a motility assay. In Figure 3 we show the trajectories of the center of mass of a single filament moving in two assays of high motor density. The persistent nature of the random walk can be clearly recognized. For higher motor density (Figure 3(b) as compared to 3(b)) the persistence length increases, and changes in the direction of motion become less frequent.

In order to check the above predictions more quantitatively we measured the mean distance between rotations $\left\langle\xi_{\text {rot }}\right\rangle$, as a function of the filament length. First, we determined $\left\langle\xi_{\text {rot }}\right\rangle$ by integrating the absolute value of the filament velocity, $\left|\partial_{t} \mathbf{r}_{i}\right|$, over time between successive rotations, see Figure 4 . It turns out that the simulation using the full model defined in section 2 gives much larger values for $\left\langle\xi_{\text {rot }}\right\rangle$ (red crosses in Figure 4) than the theoretical prediction (15) (dashed lines in Figure 4). The reason for the differences lie in a stochastic motion of 
(a)

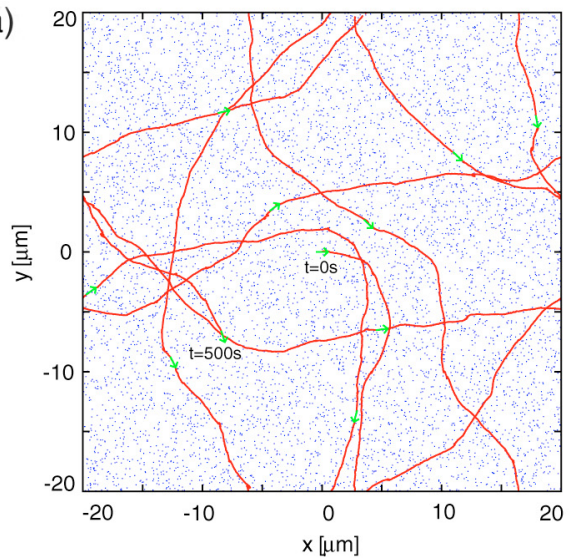

(b)

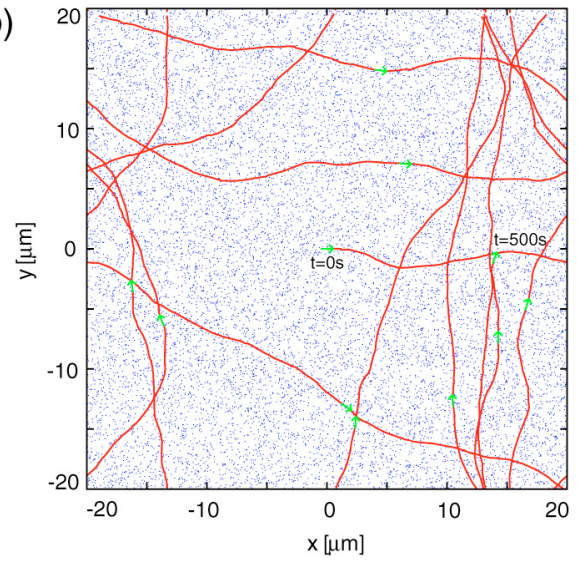

Fig. 3. Simulation trajectories (red) of a single filament of length $L=1 \mu \mathrm{m}$ (green arrow) in a gliding assay with periodic boundaries and high motor surface density, (a) $\sigma \ell_{m} L=0.44$ and (b) $\sigma \ell_{m} L=0.88$. The blue dots show the positions of individual motors. The size of the assays is $160 \mu \mathrm{m}^{2}$. The trajectories show simulation runs for 500 s, i.e., $5 \times 10^{5}$ discrete time steps. The green arrows indicate direction and position of the filament in $50 \mathrm{~s}$ intervals.

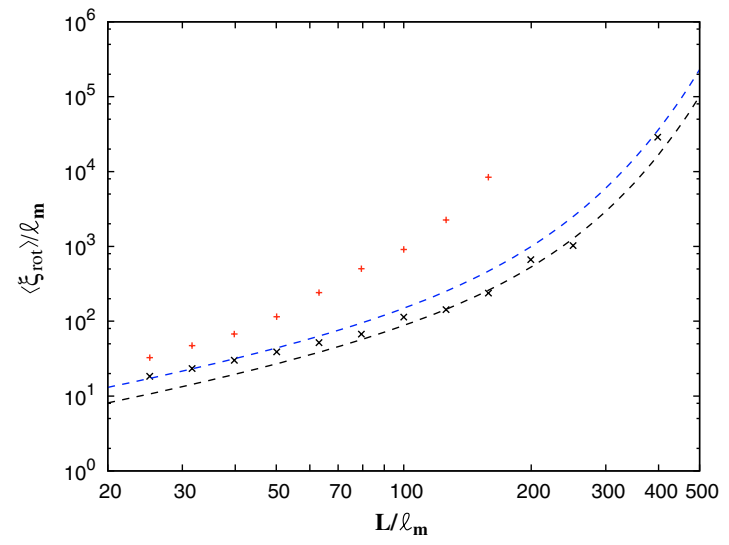

Fig. 4. Data points show simulation results for the mean distance $\left\langle\xi_{\text {rot }}\right\rangle$ traveled by a filament between successive rotations (in units of the capture radius $\ell_{m}$ ) as a function of the ratio of the filament length $L$ (in units of $\ell_{m}$ ). Red crosses are from simulations using the full model. Black crosses are results from simulations where motor and thermal forces in the direction perpendicular to the filament are suppressed as well as thermal and motor torques. The blue dotted line and black dotted line show the theoretical result from eq. (15) with $\left\langle d_{m}\right\rangle$ given by eq. (7) and $\left\langle d_{m}\right\rangle \approx 2 \sigma \ell_{m}$, respectively.

the filament perpendicular to its orientation. This fluctuating component contributes to our measurement of $\left\langle\xi_{\text {rot }}\right\rangle$ in the simulation but is not considered in the theoretical result (15). There are three mechanism, which give rise to the stochastic motion in the direction perpendicular to the filament: (i) The component of the thermal force $\boldsymbol{\zeta}_{i, \perp}$ perpendicular to $\mathbf{u}_{i}$. (ii) Every time a motor attaches or detaches the force on the filament changes discontinuously. If the filament was approximately in mechanical equilibrium before, each such force jump gives rise to a sudden filament displacement. (iii) Every time step each motor advances by $\Delta x_{i}^{\alpha}=v\left(\mathbf{F}_{i}^{\alpha}\right) \Delta t$, which leads to a small torque on the filament if it was in mechanical equilibrium before. These torques lead to a swiveling motion of the filament along its path. In order to suppress all three mechanisms motion we performed simulations where we set all motor force components $\mathbf{F}_{i}^{\alpha}$ acting on a filament $i$ in the direction perpendicular to its orientation $\mathbf{u}_{i}$ and all torques $M_{i}^{\alpha}$ to zero. We also set the corresponding components $\boldsymbol{\zeta}_{i, \perp}$ and $\zeta_{\theta, i}$ of the thermal noise to zero. 
The resulting simulation data for $\left\langle\xi_{\text {rot }}\right\rangle$ (black crosses in Figure 4) then shows good agreement with the theoretical result (15) (dashed lines in Figure 4).

In the next section we consider the phase behavior of interacting filaments in the motility assay. For our arguments the fluctuating motion in the direction perpendicular to the filament will be irrelevant because we consider time scales larger than the persistence time $t_{p}=\xi_{p} / v_{F}$ of the filament trajectory, where this noisy motion is averaged out. Therefore we use the theoretical result (15) for the persistence length in the following.

\section{Phase behavior for hard-core filament repulsion}

Now we consider the phase behavior of many interacting filaments in a motility assay. Specifically, we consider the phase behavior for a hard-core repulsion between filaments, i.e., in the limit of a large crossing energy. In the absence of motor activity, a system of rigid filaments of length $L$ represents a two-dimensional hard rod fluid, which undergoes a transition into a nematic phase at sufficiently high filament densities. Motor activity strongly modifies this nematic ordering in a motility assay. Therefore, both the rod density $\rho$ and the motor density $\sigma$ are essential in order to determine the phase behavior, which can be described in the plane of the two dimensionless parameters $\rho L^{2}$ and $\sigma \ell_{m} L$ as shown in Fig. 5. Nematic ordering in a system of $N$ filaments can be characterized by the time averages of the order parameter

$$
S \equiv \sum_{i \neq j} \cos \left(2\left(\theta_{i}-\theta_{j}\right)\right) / N(N-1) .
$$

In an infinite system, we expect $\langle S\rangle=0$ in the isotropic phase and $\langle S\rangle=1$ for perfect nematic order. In equilibrium, i.e., in the absence of motors $(\sigma=0)$ we find a continuous isotropic-nematic transition at a critical density $\rho_{c, 0} \simeq 4.3 / L^{2}$ in the simulation, which is in good agreement with the analytic result $\rho_{c, 0}=3 \pi / 2 L^{2}$ based on Onsager's theory for the two-dimensional hard-rod fluid [23]. The equilibrium transition is found numerically from the inversion point of the curve $\langle S\rangle=\langle S\rangle(\rho)$ for a value $\langle S\rangle \simeq 0.2$, which we also use as the threshold value for active nematic ordering in the presence of motors $(\sigma>0)$, see Fig. 6 . Snapshots of the actively driven system in the isotropic and nematic phase are shown in Figs. 1(a) and (b), respectively. In the resulting phase diagram Fig. 5, the critical density $\rho_{c}$ for active nematic ordering decreases with increasing motor density, i.e., nematic ordering is favored if more energy is fed into the system. The transition is continuous also for non-zero motor-density, see the order parameter plots in Fig. 6.

Each driven filament gives a contribution $\mathbf{J}_{i}=N^{-1} \rho v_{F} \mathbf{u}_{i}$ to the filament current. In the presence of motor activity and in the nematic phase, the contributions to the total filament current become correlated along a preferred direction given by the unit vector $\mathbf{n}$ leading to a non-vanishing expectation value

$$
\sum_{i \neq j}\left\langle\left(\mathbf{J}_{i} \cdot \mathbf{J}_{j}\right)^{2}\right\rangle / N(N-1)=N^{-4} \rho^{4} v_{F}^{4}\langle S\rangle .
$$

This gives rise to two macroscopic filament currents $\left\langle\mathbf{J}_{ \pm \mathbf{n}}\right\rangle= \pm \rho v_{F} \mathbf{n} / 2$ of opposite directions $\pm \mathbf{n}$ with zero total current, $0=\sum_{i}\left\langle\mathbf{J}_{i}\right\rangle=\left\langle\mathbf{J}_{\mathbf{n}}\right\rangle+\left\langle\mathbf{J}_{-\mathbf{n}}\right\rangle$. The existence of such macroscopic currents is characteristic for a non-equilibrium phase. These currents vanish (i) for small motor density, i.e., upon approaching the vertical axis $\sigma \ell_{m} L=0$ in the phase diagram Fig. 5 and (ii) upon approaching the isotropic-nematic phase boundary in the phase diagram Fig. 5, which is given by (19).

The single filament performs a persistent walk with a persistence length (15), which corresponds to a persistence time $t_{p}=\xi_{p} / v_{F}$. A coarse-graining in time by averaging over time intervals of one persistence time $t_{p}$ leads to an effective random walk of a single filament. On time scales larger than $t_{p}$ the dynamics of a filament is again described by a diffusion equation as for passive dynamics. After coarse-graining to one persistence time we can adapt the mean-field treatment of the hard-rod fluid [24] to obtain an analytical result for the phase boundary [20]. 


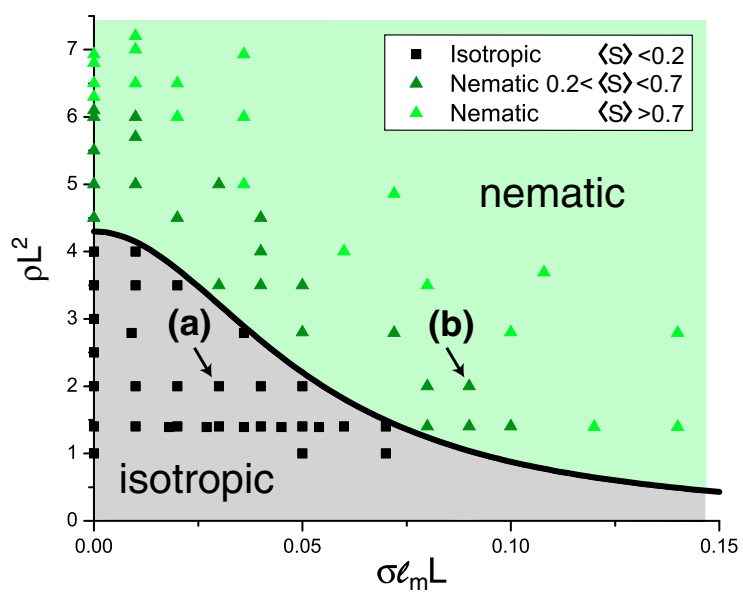

Fig. 5. The phase diagram for the gliding assay as a function of the dimensionless filament density $\rho L^{2}$ and the dimensionless surface motor density $\sigma \ell_{m} L$ for detachment force $F_{d}=F_{s t}$, and filament length $L / \ell_{m}=100$, and a hard-core repulsion between filaments. All data points correspond to separate simulation runs, the two arrows (a) and (b) to the snapshots in Fig. 1. If the average order parameter $\langle S\rangle<0.2$, the system is in the isotropic phase (black squares, grey area), if $\langle S\rangle>0.2$ it is in the nematic phase (green triangles, green area). The solid line represents the analytical result as given by (19).
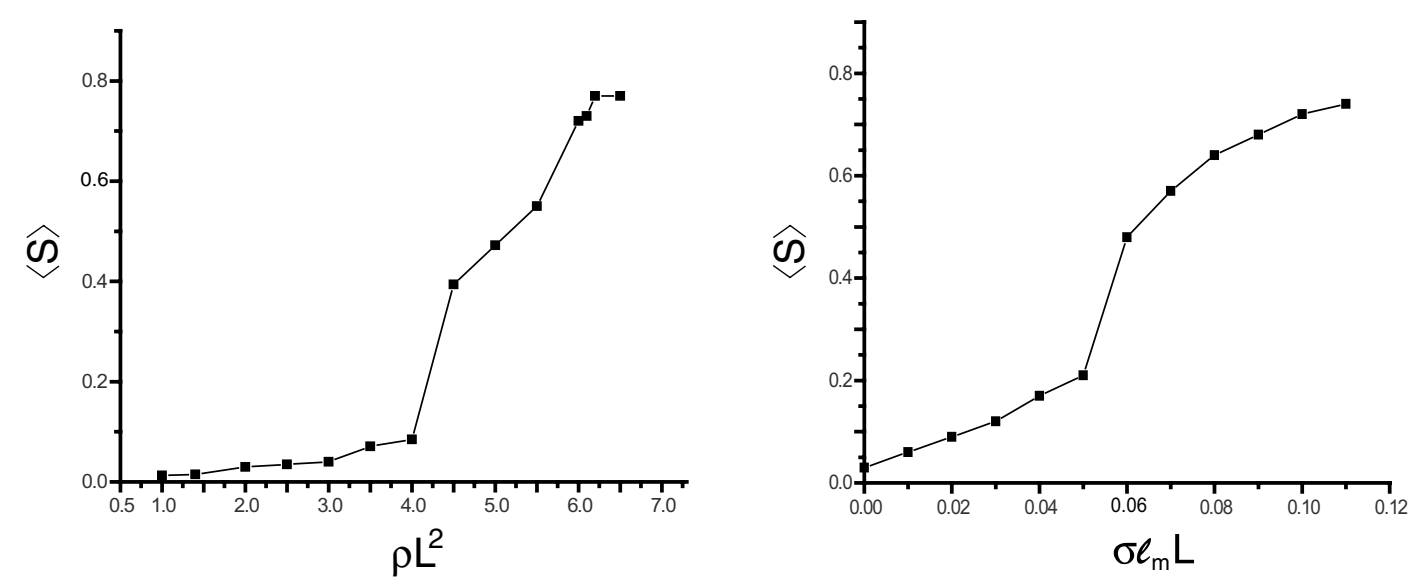

Fig. 6. Plots of the behaviour of the order parameter $\langle S\rangle$ for two trajectories in the phase diagram of Fig. 5 crossing the isotropic-nematic transition. (Left) The order parameter as a function of the dimensionless filament density $\rho L^{2}$ at zero motor density. The transition point is at $\rho L^{2}=4.3$. (Right) The order parameter as a function of the dimensionless motor density $\sigma \ell_{m} L$ for a filament density $\rho L^{2}=2$ and $L / \ell_{m}=100$ (which is the same as for the snapshots in Fig. 1 of the article). The transition point is at $\sigma \ell_{m} L=0.047$. In each plot we can identify the transition points as inflection points of the order parameter curve. Each data point in the order parameter plots corresponds to the average value of the order parameter taken over $10^{6}$ time steps.

The effective excluded area governing the steric interaction between two motor-driven filaments with center of masses and orientations $(\mathbf{r}, \mathbf{u})$ and $\left(\mathbf{r}^{\prime}, \mathbf{u}^{\prime}\right)$ on the surface of the motility assay is

$$
\begin{aligned}
A_{\mathrm{exc}} & =\left|\mathbf{u} \times \mathbf{u}^{\prime}\right| \iint_{-L / 2}^{L / 2+\xi_{p}} d \xi d \eta \Theta_{L}(\xi-\eta) \delta\left(\mathbf{r}-\mathbf{r}^{\prime}+\mathbf{u} \xi+\mathbf{u}^{\prime} \eta\right) \\
& =\left|\mathbf{u} \times \mathbf{u}^{\prime}\right| L\left(L+\xi_{p}\right),
\end{aligned}
$$

where $\Theta_{L}(\xi-\eta)$ equals one if $|\xi-\eta|<L / 2$ and zero otherwise. Performing an analogous stability analysis as in Ref. [24] using this effective excluded are we finally obtain the critical filament 
density of the active isotropic-nematic transition [21],

$$
\rho_{c}=c / L\left[L+\xi_{p}\left(\left\langle d_{m}\right\rangle, \ell_{m}, L\right)\right]
$$

with $c=3 \pi / 2$ from the analytical mean-field calculation. ${ }^{2}$

In the absence of motors we have $\xi_{p}=0$ and the result (19) reduces to the equilibrium result of Ref. [23]. Using the result (15) for the persistence length $\xi_{p}=\xi_{p}\left(\left\langle d_{m}\right\rangle, \ell_{m}, L\right)$, we obtain an explicit expression of the isotropic-nematic phase boundary in the active system in terms of the microscopic model parameters, which is in good agreement with all simulation data, see Fig. 5. Beyond mean-field, we expect a larger numerical prefactor $c$ in (19) but the same parameter dependence.

The result (19) corresponds to an effectively increased filament length $L_{\text {eff }}=\sqrt{L\left(L+\xi_{p}\right)}$ as compared to the equilibrium system, which explains that motor activity actually favors nematic ordering. In deriving the phase boundary (19), we have established a mapping of the non-equilibrium driven system onto an equilibrium system with larger effective filament length. This mapping only applies within the isotropic phase as it is based on the statistical properties of the motion of a single filament (or non-interacting filaments) but can be used to calculate the stability boundary (19) of the isotropic phase.

\section{Conclusion}

We have presented a microscopic simulation model for active filament dynamics in motility assays. First, the model is used to characterize the persistent motion of single filaments in an assay. Then, we have presented simulations and a theoretical description of the phase behavior of filaments in a two-dimensional motility assay. The corresponding equilibrium system is the two-dimensional hard-rod fluid, which exhibits an isotropic-nematic phase transition. We have found that actively driven systems undergo an analogous phase transition and the motor activity enhances the tendency for nematic ordering. A similar enhancement is found in three-dimensional active filament solutions [9]. For the two-dimensional motility assays we have quantitatively determined the phase boundary (19) for active nematic ordering by motors in terms of experimentally accessible microscopic model parameters.

This work was supported by the EC Sixth Framework Programme (as part of the STREP Active Biomics contract No. NMP4-CT-2004-516989).

\section{References}

1. J. Kierfeld, P. Gutjahr, T. Kühne, P. Kraikivski, R. Lipowsky, J. Comput. Theor. Nanosci. 3, 898 (2006)

2. D. Bray, Cell movements: from molecules to motility (Garland Publishers, New York, 2001)

3. J. Howard, Mechanics of Motor Proteins and the Cytoskeleton (Sinauer Associates, Inc., Sunderland, 2001)

4. M. Doi, S.F. Edwards, The Theory of Polymer Dynamics (Clarendon, Oxford, 1986)

5. F.J. Nédélec, T. Surrey, A.C. Maggs, S. Leibler, Nature 389, 305 (1997)

6. T. Surrey, M.B. Elowitz, P.-E. Wolf, F. Yang, F. Nédélec, K. Shokat, S. Leibler, Proc. Natl. Acad. Sci. USA 95, 4293 (1998)

7. T. Surrey, F. Nédélec, S. Leibler, E. Karsenti, Science 292, 1167 (2001)

8. H.Y. Lee, M. Kardar, Phys. Rev. E 64, 056113 (2001)

9. T.B. Liverpool, M.C. Marchetti, Phys. Rev. Lett. 90, 138102 (2003)

10. K. Kruse, J.F. Joanny, F. Jülicher, J. Prost, K. Sekimoto, Phys. Rev. Lett. 92, 078101 (2004)

\footnotetext{
2 The analogous calculation for filaments moving in a quenched array of motors in three spatial dimensions gives a critical filament density $\rho_{c}^{3 d}=c^{3 d} / D L\left[L+\xi_{p}^{3 d}\right]$ for active nematic ordering, where $\xi_{p}^{3 d}=\xi_{p} \pi^{2} L^{2} / 4 \ell_{m}^{2}$ and $c^{3 d}=4 \pi / 3$.
} 
11. I.S. Aranson, L.S. Tsimring, Phys. Rev. E 71, 050901(R) (2005)

12. F. Ziebert, W. Zimmermann, Phys. Rev. E 70, 022902 (2004)

13. D. Humphrey, C. Duggan, D. Saha, D. Smith, J. Käs, Nature 416, 413 (2002)

14. T.B. Liverpool, A.C. Maggs, A. Ajdari, Phys. Rev. Lett. 86, 4171 (2001)

15. J. Howard, A.J. Hudspeth, R.D. Vale, Nature 342, 154 (1989)

16. J. Scholey, Motility assays for motor proteins, Meth. Cell Biology 39 (Academic Press, New York, 1993)

17. T. Duke, T.E. Holy, S. Leibler, Phys. Rev. Lett. 74, 330 (1995)

18. M.R. Faretta, B. Basetti, Europhys. Lett. 41, 689 (1998)

19. F. Gibbons, J.-F. Chauwin, M. Despósito, J.V. José, Biophys. J. 80, 2515 (2001)

20. P. Kraikivski, R. Lipowsky, J. Kierfeld, Phys. Rev. Lett. 96, 258103 (2006)

21. J. Kierfeld, P. Kraikivski, R. Lipowsky, Biophys. Rev. Lett. 1, 363 (2006)

22. L. Onsager, Ann. N.Y. Acad. Sci. 51, 627 (1949)

23. R.F. Kayser, H.J. Raveché, Phys. Rev. A 17, 2067 (1978)

24. M. Doi, T. Shimada, K. Okano, J. Chem. Phys. 88, 4070 (1988); T. Shimada, M. Doi, K. Okano, J. Chem. Phys. 88, 7181 (1988)

25. C.M. Coppin, D.W. Pierce, L. Hsu, R.D. Vale, Proc. Natl. Acad. Sci. USA 94, 8539 (1997)

26. S.M. Block, C.L. Asbury, J.W. Shaevitz, M.J. Lang, Proc. Natl. Acad. Sci. USA 100, 2351 (2003)

27. J. Kierfeld, O. Niamploy, V. Sa-yakanit, R. Lipowsky, Eur. Phys. J. E 14, 17 (2004)

28. See auxiliary EPAPS Document No. E-PRLTAO-97-024627 of Ref. [20]

29. Kazuhiro Oiwa (private communication) 